\title{
BMJ Open Frontal assessment battery for detecting executive dysfunction in amyotrophic lateral sclerosis without dementia: a retrospective observational study
}

\author{
Maria Rosaria Barulli, ${ }^{1}$ Andrea Fontana, ${ }^{2}$ Francesco Panza, ${ }^{1,3}$ \\ Massimiliano Copetti, ${ }^{2}$ Stefania Bruno, ${ }^{1}$ Marianna Tursi, ${ }^{3}$ Annalisa lurillo, ${ }^{3}$ \\ Rosanna Tortelli, ${ }^{1}$ Rosa Capozzo, ${ }^{3}$ Isabella Laura Simone, ${ }^{3}$ \\ Giancarlo Logroscino ${ }^{1,3}$
}

To cite: Barulli MR, Fontana A, Panza F, et al. Frontal assessment battery for detecting executive dysfunction in amyotrophic lateral sclerosis without dementia: a retrospective observational study. BMJ Open 2015;5:e007069. doi:10.1136/bmjopen-2014007069

- Prepublication history and additional material is available. To files please visit the journal (http://dx.doi.org/ 10.1136/bmjopen-2014007069).

Received 31 October 2014 Revised 22 May 2015 Accepted 13 June 2015

CrossMark

For numbered affiliations see end of article.

Correspondence to Dr Giancarlo Logroscino; giancarlo.logroscino@uniba.it

\section{ABSTRACT}

Objective: The frontal assessment battery (FAB) is a quick and reliable method of screening to evaluate frontal lobe dysfunction in amyotrophic lateral sclerosis (ALS). However, previous studies were generally conducted on small samples representing different stages of disease and severity. We assessed the diagnostic accuracy of the FAB in detecting executive functions and its association with demographic and clinical features in ALS without dementia.

Design: Retrospective observational study.

Setting: A multidisciplinary tertiary centre for motor neuron disease.

Participants: We enrolled 95 consecutive patients with ALS diagnosed with El Escorial criteria in the period between January 2006 and December 2010.

Main outcome measures: We screened the patients with ALS using the FAB. An Executive Index (EI) was also calculated by averaging the $Z$ scores of analytic executive tests evaluating information-processing speed (Symbol Digit Modalities Test-Oral version), selective attention (Stroop test) and semantic memory (Verbal Fluency Test).

Results: The FAB detected executive dysfunction in $13.7 \%$ of the patients with ALS. Moreover, using the El standardised cut-off, $37.9 \%$ of the patients with ALS showed executive dysfunction. The receiver-operating characteristic curve showed that the optimal cut-off for the $F A B$ in the whole sample was 16 , with a sensitivity of 0.889 (95\% Cls 0.545 to 1.000$)$, a specificity of $0.593(95 \% \mathrm{Cl} 0.450$ to 0.907$)$ and a moderate overall discriminatory power of 0.809 . Different levels of respiratory function, duration of disease and depressive symptoms did not affect the FAB validity.

Conclusions: In patients with ALS without dementia, a high prevalence of executive dysfunction was present. The FAB showed good validity as a screening instrument to detect executive dysfunction in these patients and may be used when a complete neuropsychological assessment is not possible.

\section{Strengths and limitations of this study}

- The strengths of this work include relatively stringent criteria for the definition of cognitive impairment, the use of gold standard tests to assess executive dysfunction and a larger sample of patients, as compared with previous studies.

- To the best of our knowledge, this is the first study in which a tree-based method has been used to evaluate the influence of various demographic and clinical variables on executive dysfunction in amyotrophic lateral sclerosis.

- A limitation is that our sample was clinically heterogeneous, including patients with an unusually long duration of disease and low disability.

- The small number of participants in the final RECursive Partitioning and AMalgamation (RECPAM) analysis, together with the wide SDs, raises the possibility that a larger sample may improve the performance of the frontal assessment battery in more clinically defined subgroups.

- The cognitive screening we adopted was limited to executive functions, and it is possible that the dysfunction of more posterior regions of the brain could go undetected.

\section{INTRODUCTION}

Amyotrophic lateral sclerosis (ALS) is a neurodegenerative illness previously described as a motor disease, but the presence of more complex phenotypes including cognitive and behavioural features is now widely accepted. Cognitive impairment may manifest at the same time as the motor symptoms, or even precede them, ${ }^{1}{ }^{2}$ with different degrees of severity, ranging from milder cognitive dysfunction to overt dementia, generally with a 
frontotemporal pattern. ${ }^{3}$ The presence of cognitive dysfunction in patients with ALS determines a worse prognosis in terms of life expectancy. ${ }^{4}$

Few preliminary studies using a screening battery to detect cognitive impairment in ALS have indicated that a brief cognitive screening of these patients can be easily performed in clinical outpatient settings. ${ }^{5}$ Since cognitive deficits in ALS are largely of executive functioning, the use of a screening tool designed for the detection of frontal dysfunction may be a possible advantageous option.

The frontal assessment battery $(\mathrm{FAB})^{6} 7$ is a short and easily administered cognitive test that has been shown to be better than the Mini-Mental State Examination $(\mathrm{MMSE})^{8}$ as a screening tool in neurodegenerative disease with frontal involvement. Few studies have used the $\mathrm{FAB}^{9-11}$ to evaluate frontal lobe dysfunction in ALS, and their findings suggested that the FAB is a quick and reliable method of screening. However, these studies used generally small samples representing different stages of disease and severity. The aims of the present study were to detect executive dysfunction using the FAB in a large sample of patients with ALS without dementia and to assess the diagnostic accuracy of the FAB compared with a standardised extensive neuropsychological assessment of executive functions. Finally, we investigated the association between impairment of executive functions and demographic and clinical features of ALS.

\section{METHODS}

\section{Participants}

This study was conducted according to the World Medical Association's 2008 Declaration of Helsinki and the guidelines for Good Clinical Practice and the Strengthening the Reporting of Observational Studies in Epidemiology (STROBE) statement ${ }^{12}$ and STAndards for the Reporting of Diagnostic accuracy (STARD) studies. ${ }^{13}$ In this retrospective cohort study, we enrolled consecutive patients with ALS attending a specialised multidisciplinary centre for motor neuron disease at the University of Bari 'Aldo Moro' in the period between January 2006 and December 2010.

The diagnosis of ALS was based on El Escorial criteria (EEC). ${ }^{14}$ Patients who had a dementia syndrome according to the Diagnostic and Statistical Manual of Mental Disorders IV Text Revision, ${ }^{15}$ and participants with an MMSE score lower than 26 were excluded. ${ }^{16}$ Participants with extreme weakness of the hands and dysarthria were excluded because they were unable to perform the tests. We did not include patients for whom one or more of the neuropsychological tests were unavailable. All the patients with ALS were taking riluzole when they were interviewed for this study. Functional impairment at the neurological examination was evaluated using the revised ALS Functional Rating Scale-revised (ALSFRS-R) ${ }^{17}$ and manual muscle testing (MMT). ${ }^{18}$ Respiratory function was measured by forced vital capacity (FVG). ${ }^{19}$

\section{Neuropsychological assessment}

The patients underwent a global screening of executive functions using the Italian version of the FAB. ${ }^{6} 7$ This consisted of six subset test items: conceptualisation (abstract reasoning), item flexibility (verbal fluency), motor programming (organisation, maintenance and execution of successive actions), sensitivity to interference (conflicting instructions), inhibitory control (inhibit inappropriate responses), and environmental autonomy (prehension behaviour). The administration time of the FAB is about $10 \mathrm{~min}$. Some executive features were also evaluated using three tests commonly used in clinical practice. The first domain was the information-processing speed, possibly determined by attentional capacity (scanning and tracking in the visuospatial domain) and working memory, assessed with the Symbol Digit Modalities Test (SDMT)-Oral version. ${ }^{20}{ }^{21}$ Furthermore, cognitive interference in processing incongruent information preventing habitual or automatic responses was assessed with the Stroop test (ST). ${ }^{22}$ Finally, the ability to select an appropriate research strategy in lexical access and retrieval was assessed with the Verbal Fluency Test (VFT). ${ }^{23}{ }^{24}$ The Beck Depression Inventory (BDI) ${ }^{25}$ was administered to evaluate the presence of depressive symptoms. Patients with a BDI score $>10$ were considered depressed. Two trained neuropsychologists administered to the patients with ALS the FAB or the neuropsychological executive test battery and were blind to the results of other tests.

\section{Statistical analysis}

Demographic and clinical characteristics of the patients were reported as frequency (percentages) and mean \pm SD for categorical variables and continuous variables, respectively. Standardised $\mathrm{Z}$ scores were calculated for all the executive tasks, based on Italian normative data. A cut-off of 2 SD below the mean for standardised data was used to define an abnormal cognitive performance on any task. An ad hoc Executive Index (EI) was defined for each patient by averaging the $\mathrm{Z}$ scores of SDMT, the ST items and VFT executive tasks. Patients were categorised as impaired if they had an EI of 2 SD below the mean. Since EI was defined as the mean of four standardised random variables, the computed cut-off of 2 SD below the mean was -1 . Correlations between the FAB (total score and its subset of items), EI and the executive tests (SDMT, ST and VFT) were assessed by the Spearman coefficients. Correlations between the FAB total score, EI and demographic and clinical variables were also assessed by the Spearman coefficients. A tree-growing technique, based on the RECursive Partitioning and AMalgamation (RECPAM) algorithm, ${ }^{26}$ was used to investigate possible interactions among demographic and clinical variables in order to identify distinct and homogeneous patient subgroups in terms of EI. At each partitioning step, the RECPAM method selects the covariate and the best binary split that maximises the difference of EI means between 
subgroups, following a linear regression model. Age, sex, ALS diagnostic level based on EEC, site of the onset (spinal vs bulbar), education level, disease duration, the degree of disability, BDI, FVC and the mean of MMT were considered as candidate splitting variables. To obtain more robust splits, a bootstrap approach with 100000 node resampling with replacement was performed. The algorithm stopped when there were less than 14 participants (ie, the minimum leaf size, about $15 \%$ of the total sample) within each terminal node (user defined stopping rule). The discriminatory power of the FAB (total score) to detect the patient's executive dysfunction (defined by the EI) was assessed by estimating the Area Under the receiver operating characteristic Curve (AUC) ${ }^{27}$ and was evaluated both in the overall sample and within each RECPAM class. The optimal cut-off for the FAB was assessed by maximising jointly sensitivity and specificity. Non-parametric bootstrap $95 \%$ CIs for optimal specificity and sensitivity were estimated at the optimal cut-off point using 1000 bootstrap samples. Specifically, CIs were derived by using the 2.5 and 97.5 centiles of the bootstrap distribution as the limits of the $95 \%$ CI. This approach provided more robust, conservative and validated results. ${ }^{28} \mathrm{~A} \quad \mathrm{p}$ value $<0.05$ was considered for statistical significance. All analyses were performed using SAS Release 9.1.3 (SAS Institute, Cary, North Carolina, USA).

\section{RESULTS}

We enrolled a total of 152 consecutive patients with ALS. We excluded 57 patients: 4 patients because of dementia syndrome, 32 due to MMSE $<26$, 4 for missing neuropsychological tests, and 17 due to weakness in the hands and dysarthria. Therefore, 95 patients with ALS (62 males and 33 females, age range 33-82 years) were included in the analyses. Demographic and clinical data are reported in table 1 . Means and SD for the FAB, SDMT, VFT and ST were, respectively, 15.51 $\pm 2.74,31.75$ $\pm 13.23,25.94 \pm 9.60$ and $27.54 \pm 14.92$. Executive dysfunction was detected in $88.4 \%$ of the patients $(\mathrm{n}=84)$ with the VFT, $77.9 \% \quad(\mathrm{n}=74)$ with the ST and $35.1 \% \quad(\mathrm{n}=33)$ with the SDMT. Using the EI standardised cut-off, $37.9 \%$ of the patients $(\mathrm{n}=36)$ showed executive dysfunction. The FAB detected executive dysfunction in 13.7\% $(n=13)$ of the patients (cut-off score: 13.5). The FAB total score was significantly correlated with EI $(r=0.667$, $\mathrm{p}<0.001)$ and with each component of EI: SDMT $(\mathrm{r}=0.610, \mathrm{p}<0.001)$, VFT $(\mathrm{r}=0.594, \mathrm{p}<0.001)$ and ST $(\mathrm{r}=$ $-0.434, \mathrm{p}<0.001)$, showing high concurrent validity. Furthermore, the FAB subset-item scores were significantly correlated with the other executive function tests (table 2). A substantial presence of depressive symptoms (BDI score $>10$ ) was detected in $45.26 \%$ of the patients with ALS $(n=43)$.

Both the FAB total score and EI correlated with age and respiratory function, while only the FAB total score correlated with disease duration (table 3). Furthermore,
Table 1 Demographic and clinical parameters of 95 patients with ALS without dementia

\begin{tabular}{|c|c|}
\hline Variables & $\begin{array}{l}\text { Number }(\%) \text { or mean } \pm \text { SD } \\
\text { Median (range) }\end{array}$ \\
\hline Age, in years & $\begin{array}{l}61.22 \pm 10.66 \\
62(33-82)\end{array}$ \\
\hline \multicolumn{2}{|l|}{ Sex } \\
\hline Males & $62(65.26 \%)$ \\
\hline Females & $33(34.74 \%)$ \\
\hline Education years & $\begin{array}{l}9.23 \pm 3.86 \\
8.00(0-18)\end{array}$ \\
\hline \multicolumn{2}{|l|}{ ALS diagnosis } \\
\hline Definite & $33(34.73 \%)$ \\
\hline Probable & 36 (37.89\%) \\
\hline Possible & $16(16.84 \%)$ \\
\hline Suspect & $10(10.52 \%)$ \\
\hline \multicolumn{2}{|l|}{ Site of onset } \\
\hline Spinal & 85 (89.47\%) \\
\hline Bulbar & $10(10.52 \%)$ \\
\hline Time to diagnosis (months) & $\begin{array}{l}16.91 \pm 22.99 \\
10.00(0.34-120)\end{array}$ \\
\hline Disease duration (months) & $\begin{array}{l}29.09 \pm 30.94 \\
18.00(2-148)\end{array}$ \\
\hline FVC & $\begin{array}{l}86.58 \pm 23.33 \\
91.50(32.4-131.6)\end{array}$ \\
\hline MMT & $\begin{array}{l}8.33 \pm 1.43 \\
8.60(2.7-10)\end{array}$ \\
\hline ALSFRS-R & $\begin{array}{l}37.29 \pm 7.29 \\
39.00(7-48)\end{array}$ \\
\hline BDI & $\begin{array}{l}10.60 \pm 7.49 \\
10(0-29)\end{array}$ \\
\hline
\end{tabular}

we analysed the correlation between the FAB and EI with different levels of respiratory function, disease duration and depressive symptoms. Stratified analysis by respiratory function (FVC: $\leq 80$ vs $>80$ ) showed a positive correlation between the FAB and EI in low $(r=0.559$, $\mathrm{p}<0.001)$ and high respiratory function subgroups $(\mathrm{r}=0.731, \mathrm{p}<0.001)$. Similarly, the analysis by duration of illness ( $\leq 18$ vs $>18$ months, median value) showed a positive correlation between the FAB and EI in short $(\mathrm{r}=0.601, \mathrm{p}<0.001)$ and long disease duration subgroups $(\mathrm{r}=0.732, \mathrm{p}<0.001)$. When we considered the depressive symptoms in patients with a disease duration of less than 5 years $(n=81)$, the FAB total score was correlated with the tests of EI in both groups depressed $(n=47$; EI $\mathrm{r}=0.740, \mathrm{p}<0.001$; SDMT $\mathrm{r}=0.773, \mathrm{p}<0.001$; VFT $\mathrm{r}=0.756$, $\mathrm{p}<0.001$; ST $\mathrm{r}=-0.435, \mathrm{p}=0.002)$ versus not depressed $(\mathrm{n}=34$; EI $\mathrm{r}=0.576, \mathrm{p}<0.001$; SDMT $\mathrm{r}=0.443, \mathrm{p}=0.009$; VFT $\mathrm{r}=0.320, \mathrm{p}=0.065$; $\mathrm{ST} \mathrm{r}=-0.479, \mathrm{p}=0.004)$. The correlation between the FAB and executive function tests was also present when we restricted the analysis to incident cases $(\mathrm{n}=35)$ with disease duration $<12$ months (EI $\mathrm{r}=0.665$, $\mathrm{p}<0.001$; SDMT $\mathrm{r}=0.549, \mathrm{p}=0.001$; VFT $\mathrm{r}=0.526, \mathrm{p}=0.001$; ST $r=-0.448, p=0.007)$. 
Table 2 Spearman correlation coefficients between the FAB and executive function tests (raw values) in patients with ALS without dementia

\begin{tabular}{lllr}
\hline FAB subitems & SDMT & VFT & ST \\
\hline Conceptualisation & $0.473(p<0.001)$ & $0.361(p<0.001)$ & $-0.302(p=0.003)$ \\
Mental flexibility & $0.457(p<0.001)$ & $0.730(p<0.001)$ & $-0.297(p=0.004)$ \\
Motor programming & $0.304(p=0.003)$ & $0.174(p=0.092)$ & $-0.0508(p=0.625)$ \\
Sensitivity to interference & $0.317(p=0.002)$ & $0.286(p=0.005)$ & $-0.221(p=0.031)$ \\
Inhibitory control & $0.256(p=0.012)$ & $0.275(p=0.007)$ & $-0.370(p<0.001)$ \\
Environmental autonomy & $0.108(p=0.296)$ & $0.106(p=0.308)$ & $0.021(p=0.837)$ \\
FAB total score & $0.610(p<0.001)$ & $0.594(p<0.001)$ & $-0.434(p<0.001)$ \\
\hline ALS, amyotrophic lateral sclerosis; FAB, frontal assessment battery; SDMT, Symbol Digit Modalities Test; ST, Stroop Test; VFT, Verbal \\
Fluency Test.
\end{tabular}

Results of the RECPAM analysis are shown in figure 1. The algorithm identified four homogeneous subgroups of patients in terms of EI means (classes 1-4). Class 1 represented the patient subgroup with the lowest standardised mean EI (and thus with the highest executive cognitive impairment), whereas class 4 represented the patient subgroup with the highest standardised mean EI (and thus with the lowest executive cognitive impairment). Specifically, the algorithm found that patients with an education level $\leq 8$, age $>58$ years and FVC $\leq 86.4$ represented the class (class 1) with the lowest standardised mean EI (ie, $-2.92 \pm 2.67, \mathrm{~N}=20$ patients). Patients with an education level $\leq 8$, age $>58$ years and $\mathrm{FVC}>86.4$ represented the class (class 2) with a quite lower standardised mean EI (ie, $-1.45 \pm 1.17, \mathrm{~N}=20$ patients). Patients with an education level $\leq 8$ and age $\leq 58$ years represented the class (class 3) with a higher standardised mean EI (ie, $-0.87 \pm 1.24, \mathrm{~N}=20$ patients), and finally patients with an education level $>8$ represented the class (class 4) with the highest standardised mean EI (ie; $-0.14 \pm 0.71, \mathrm{~N}=35$ patients). Furthermore, using the EI standardised cut-off to identify patients with cognitive impairment (gold standard), the optimal cut-off for the FAB (total score) was detected both in the whole sample and within each identified RECPAM class. The receiveroperating characteristic (ROC) curve showed that the optimal cut-off for the FAB in the whole sample was 16

Table 3 Correlation between demographic, clinical and cognitive variables in patients with ALS without dementia

\begin{tabular}{|c|c|c|c|c|}
\hline Age & $\begin{array}{l}\text { Disease } \\
\text { duration }\end{array}$ & FVC & MMT & ALSFRS-R \\
\hline \multicolumn{5}{|l|}{ El } \\
\hline $\begin{array}{l}r=-0.482 \\
p<0.001\end{array}$ & $\begin{array}{l}r=-0.055 \\
p=0.603\end{array}$ & $\begin{array}{l}r=0.354 \\
p<0.001\end{array}$ & $\begin{array}{l}r=0.048 \\
p=0.656\end{array}$ & $\begin{array}{l}r=0.094 \\
p=0.379\end{array}$ \\
\hline \multicolumn{5}{|l|}{$F A B$} \\
\hline $\begin{array}{l}r=-0.379 \\
p<0.001\end{array}$ & $\begin{array}{l}r=-0.213 \\
p=0.040\end{array}$ & $\begin{array}{l}r=0.234 \\
p=0.024\end{array}$ & $\begin{array}{l}r=0.130 \\
p=0.223\end{array}$ & $\begin{array}{l}r=0.161 \\
p=0.127\end{array}$ \\
\hline
\end{tabular}

ALS, amyotrophic lateral sclerosis; ALSFRS-R, ALS Functional Rating Scale-revised; El, Executive Index; FAB, frontal assessment battery; FVC, forced vital capacity; MMT, manual muscle testing. (figure 2). Such a cut-off achieved a high sensitivity of 0.889 (95\% CI 0.545 to 1.000 ), a low specificity of 0.593 (95\% CI 0.450 to 0.907 ), a positive predictive value (PPV) of 0.571 (95\% CI 0.446 to 0.698 ) and a negative predictive value (NPV) of $0.897 \quad(95 \%$ CI 0.794 to 0.977). The overall discriminatory power (AUC) for the FAB was 0.809. The optimal cut-off for the FAB in patients within each RECPAM class was also assessed. Results are reported in online supplementary figure S1 and in the appendix.

\section{Power calculation}

A sample of 36 patients with executive dysfunction and 59 patients without executive dysfunction achieved $90 \%$ power to detect an overall discriminatory power (AUC) for the FAB (total score) of 0.693, under the null hypothesis of AUC of 0.50 , using a two-sided z-test at a significance $(\alpha)$ level of 0.05 .

\section{DISCUSSION}

In this study, the FAB showed good validity as a screening instrument to detect executive dysfunction in patients with ALS without dementia and to select participants who will undergo a full neuropsychological examination in tertiary centres. The FAB may be especially useful in settings where a complete neuropsychological assessment cannot be proposed because of lack of time or insufficient neuropsychological staff. Respiratory function, disease duration and depressive symptoms did not affect the screening ability of the FAB to detect executive dysfunction. In fact, the correlation between the FAB and other executive tests was present in patients in low and high respiratory function subgroups, assessed in both the early and late stages of disease, and in both depressed and not depressed patients with long disease duration. The assessment of executive functioning showed a high prevalence of cognitive impairment in the executive domain with about $40 \%$ of patients with ALS showing executive dysfunction detected with EI.

In this study, the FAB cut-off score of 16 showed an AUC of 0.81 , which, according to Swets ${ }^{29}$ classification $(0.7<$ AUC $\leq 0.9)$, indicated moderate accuracy. Within each RECPAM class, the AUC ranged from 0.64 in class 
Figure 1 RECPAM tree. The sample was divided into distinct and homogeneous patients' subgroups in terms of El. Splitting variables are shown between branches. The condition sending patient to the right or left is shown on the corresponding branch.

Class 4, with the highest El mean, represents the reference class. Circles indicate subgroups of patients. Squares indicate the patient subgroup RECPAM class. Numbers inside circles and squares represent the number of participants within each class (EI, Executive Index; FVC, forced vital capacity; RECPAM, RECursive Partitioning and AMalgamation).

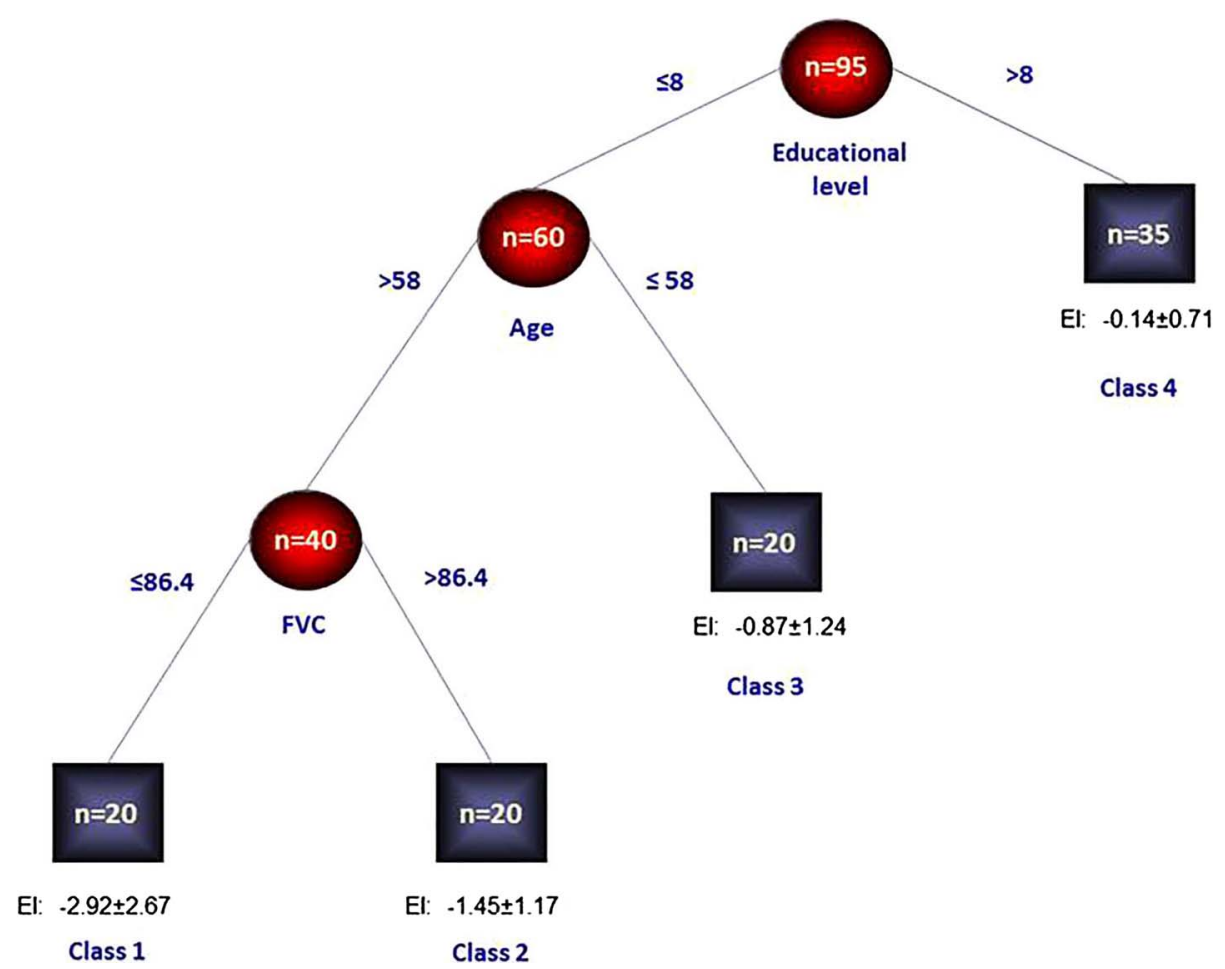

I with the highest executive cognitive impairment (cut-off score of 16.5) to 0.82 in class 4 with the lowest executive cognitive impairment (cut-off score of 17.5). The optimal FAB cut-off score of 16 for our population is higher than the standard FAB cut-off score for the Italian normative data (13.5), ${ }^{7}$ reflecting the milder spectrum of executive dysfunction in these patients. We excluded patients with dementia and with serious

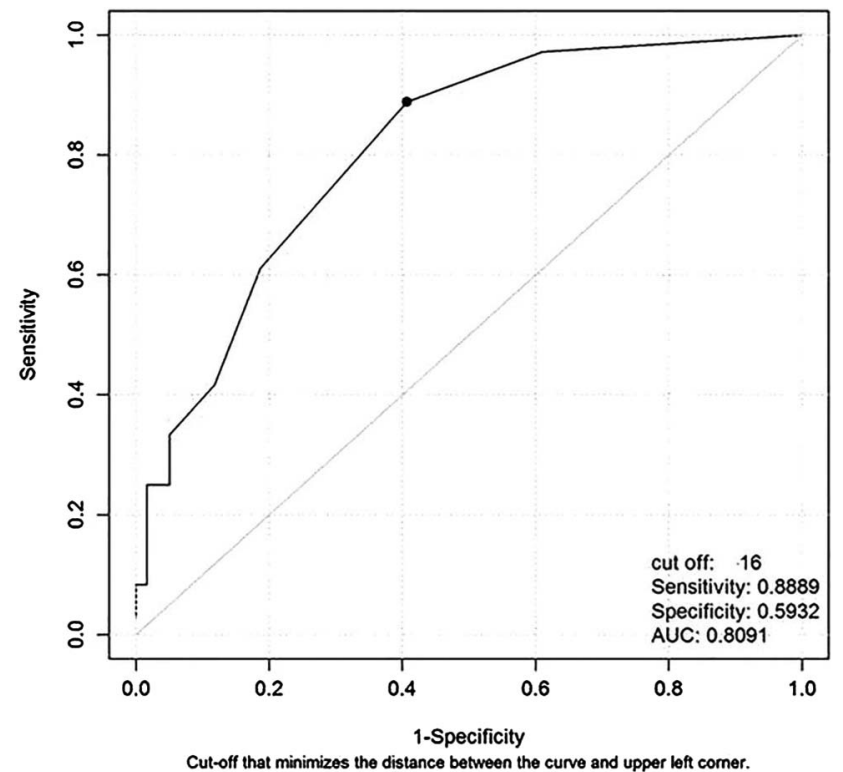

Figure 2 ROC curve for the frontal assessment battery index to detect patients with executive dysfunction evaluated in the whole sample (AUC, Area Under the receiver operating characteristic Curve; ROC, receiver-operating characteristic). impairment of the upper limbs. It was also confirmed that executive dysfunction was positively associated with age and respiratory dysfunction, but not with other clinical variables, including measures of functional status. Therefore, age level may have significant effects on the FAB performance in ALS, suggesting that the age of the participants should be taken into consideration in detecting executive dysfunction with this cognitive tool. Furthermore, as reported by other studies, no significant association was found between executive dysfunction and functional status, implying that cognitive impairment was independent from progression of the motor disability and overall disease severity. ${ }^{30}{ }^{31}$ Forty-five per cent of our sample had depressive symptoms, confirming how depression may be a common feature in patients with ALS. ${ }^{32-34}$

The results of this study are overall in keeping with earlier findings, although the majority of previous Oskarsson et at reported an impaired FAB performance in $50 \%$ of patients with ALS correlated with disease duration but not with severity of disease, based on a sample size of 16 patients without independent cognitive assessment. A Korean study showed FAB scores indicating executive dysfunction in $22.9 \%$ of 61 patients with ALS, associated with disease duration and severity of illness, but with the important limitation of using Italian normative data applied to a Korean population. ${ }^{10}$ Floris et $a l^{11}$ demonstrated that the FAB has good validity, even in early stages of the disease, using a full neuropsychological battery as the gold standard. However, the sample size was very small $(n=20)$ and correlations with clinical features in ALS were not explored. Another recent studies were based on smaller samples. ${ }^{9-11} \quad 35 \quad 36$ 
Dutch study suggested that the FAB should be administered to patients without moderate or severe speech and motor impairment. ${ }^{35}$ Finally, the FAB and the Montreal Cognitive Assessment (MoCA) were used to screen frontal lobe and general cognitive impairment in 44 patients with ALS; and while the MoCA classified more patients as cognitively impaired, the FAB was more feasible for assessing patients with physical impairment. Unfortunately, no optimal cut-off scores for these two screening instruments were provided. ${ }^{36}$

The cognitive phenotype in ALS has not yet been completely defined. At present, the most accredited hypothesis is that in a subgroup of patients with ALS there is early involvement of executive functions with later involvement of other cognitive domains. There are patients, in contrast, who show no signs of cognitive impairment throughout the entire course of the disease. ${ }^{31}$ The variety of neuropsychological instruments used for the assessments $^{3}{ }^{31}$ may be responsible for another piece of the described variability. However, the heterogeneity may be related to the presence of distinct ALS cognitive phenotypes since the early stage of disease. Our study confirms that executive dysfunction may represent a specific cognitive phenotype in a subgroup of patients with ALS. The clinical evidence of overlap between frontotemporal dementia (FTD) and motor neuron disease ${ }^{37-39}$ has recently gained further support after the C9ORF72 mutation was found to be the most common genetic abnormality in familial and sporadic forms of FTD and ALS, particularly frequent in patients and families with both conditions. ${ }^{40-42}$ Given the high prevalence of cognitive impairment and behavioural changes in ALS, the evaluation of neuropsychological abilities should be a fundamental part of the multidisciplinary initial assessment for every new patient. ${ }^{3}$ Extensive neuropsychometry is laborious and requires trained staff explaining why cognitive evaluation as part of the initial assessment is carried out in a limited number of ALS centres. In this study, executive dysfunction, as measured by the EI, assessing primarily access and retrieval lexical and cognitive interference, was present in about one-third of patients without dementia. This is in agreement with other studies that showed that executive dysfunction is the most prominent cognitive deficit in patients with ALS. ${ }^{3}{ }^{38}$ In this study, none of the patients in whom the FAB and subsequent full cognitive evaluations indicated the presence of a cognitive impairment had spontaneously reported cognitive dysfunction during the clinical interview. This probably reflects the different relevance of cognitive impairment compared with motor symptoms for the life of patients in the early phases of disease.

There are a number of limitations in this study. Our sample was enrolled in a tertiary centre that is similar to other referral centres for these main characteristics: long disease duration, less functional impairment, relatively younger patients, and a higher prevalence of spinal onset compared with bulbar onset. Furthermore, the retrospective design of this study may have several pitfalls, including the lack of assessment of certain prognostic factors, such as symptoms progression rate, the less-accurate diagnosis usually based only on the retrospective revision of clinical data, and the risk of missing specific subsets of patients with ALS not captured by the study design. Our sample was also clinically heterogeneous including patients with an unusually long duration of disease and low disability. Other possible sources of heterogeneity such as psychosocial factors (ie, perceived stress, marital status or anger expression) or malnourishment, a relevant determinant of cognitive function in ALS, have not been addressed in this study. The small number of participants in the final RECPAM analysis, together with the wide SDs, raises the possibility that a larger sample may improve the performance of the $\mathrm{FAB}$ in more clinically-defined subgroups. Finally, the cognitive screening we adopted was limited to executive functions, and it is possible that dysfunction of more posterior regions could go undetected. The strengths of this work include relatively stringent criteria for the definition of cognitive impairment, the use of gold standard tests to assess executive dysfunction and a larger sample of patients, as compared with previous studies. ${ }^{9-11} 303435$ Also, to the best of our knowledge, this is the first time that a tree-based RECPAM analysis (a generalisation of Classification And Regression Tree (CART)) has been used to study the influence of various demographic and clinical variables on cognitive dysfunction in ALS. Tree-based methods are well-established techniques to address interactions detection and subgroup analyses, as well as to derive internally validated cut-off for continuous predictors which could not be necessarily linear.

In conclusion, our findings demonstrated that the FAB had good diagnostic accuracy for detecting executive dysfunction in a population of patients with ALS without dementia and severe impairment of upper limbs referred to a tertiary centre. It should be stressed that the use of such screening instruments should not preclude a complete neuropsychological evaluation focused on executive functions, as well as on other cognitive domains such as language and visuospatial abilities. In patients with ALS, a recent and suitable cognitive tool to screen all cognitive domains is the Edinburgh Cognitive and Behavioural ALS Screen (ECAS). ${ }^{43}$ However, the ECAS was designed to detect the profile of cognitive and behavioral changes in ALS and was not specifically focused on executive functions. In fact, the ECAS evaluates five cognitive (social cognition, language, fluency, memory, and visuospatial functions), and five behavioural domains, characterising FTD. ${ }^{42}$ With the recent emphasis on the genetic and clinical overlap between motor neuron disease and FTD, systematic cognitive screening of patients with ALS would facilitate longitudinal studies that are much needed to clarify the different clinical patterns of disease, including prognosis and their different biological substrates. 
Author affiliations

${ }^{1}$ Department of Clinical Research in Neurology, Neurodegenerative Disease Unit, University of Bari Aldo Moro, "Pia Fondazione Cardinale G. Panico", Tricase, Lecce, Italy

${ }^{2}$ Unit of Biostatistics, IRCCS Casa Sollievo della Sofferenza, San Giovanni Rotondo, Foggia, Italy

${ }^{3}$ Department of Basic Medicine, Neuroscience, and Sense Organs, University of Bari Aldo Moro, Bari, Italy

Contributors MRB, FP and GL contributed to the concept of the study and are guarantors. MRB, FP and GL also contributed to the interpretation of the data and coordinated the manuscript preparation. AF, MC and GL completed the statistical analysis. SB, Al, RT, RC and ILS contributed to further interpretations and commenting on drafts of the manuscript in its preparation. MRB, GL, FP, AF and MC had full access to all of the data in the study. All authors contributed to the interpretation of the results, read and commented on the manuscript, and approved the final version.

Funding This research was in part supported by the European Community's Seventh Framework Programme (FP7/2007-2013 under grant agreement 259867).

Competing interests None declared.

Patient consent Obtained.

Ethics approval This study was approved by the Ethic Committee of 'Azienda Ospedaliera, Ospedale Policlinico Consorziale, Bari, Italy'.

Provenance and peer review Not commissioned; externally peer reviewed.

Data sharing statement No additional data are available.

Open Access This is an Open Access article distributed in accordance with the Creative Commons Attribution Non Commercial (CC BY-NC 4.0) license, which permits others to distribute, remix, adapt, build upon this work noncommercially, and license their derivative works on different terms, provided the original work is properly cited and the use is non-commercial. See: http:// creativecommons.org/licenses/by-nc/4.0/

\section{REFERENCES}

1. Strong MJ, Grace GM, Orange JB, et al. A prospective study of cognitive impairment in ALS. Neurology 1999;53:1665-70.

2. Lomen-Hoerth C, Murphy J, Langmore S, et al. Are amyotrophic lateral sclerosis patients cognitively normal? Neurology 2003;60:1094-7.

3. Strong MJ, Grace GM, Freedman M, et al. Consensus criteria for the diagnosis of frontotemporal cognitive and behavioural syndromes in amyotrophic lateral sclerosis. Amyotroph Lateral Scler 2009;10:131-46.

4. Elamin M, Phukan J, Bede $P$, et al. Executive impairment is a negative prognostic indicator in ALS patients without dementia. Neurology 2011;76:1263-9.

5. Gordon PH, Wang Y, Doorish C, et al. A screening assessment of cognitive impairment in patients with ALS. Amyotroph Lateral Scler 2007;8:362-5.

6. Dubois B, Slachevsky A, Litvan I, et al. The FAB: Frontal Assessment Battery at bedside. Neurology 2000;55:1621-6.

7. Apollonio I, Leone $\mathrm{M}$, Isella $\mathrm{V}$, et al. The frontal assessment battery (FAB): normative values in an Italian population sample. Neurol Sci 2005;26:108-16.

8. Folstein MF, Folstein SE, McHugh P. 'Mini-mental state': a practical method for grading the cognition of patients for the clinician. $J$ Psychiatric Res 1975;12:189-98.

9. Oskarsson B, Quan D, Rollins YD, et al. Using the Frontal Assessment Battery to identify executive function impairments in amyotrophic lateral sclerosis: a preliminary experience. Amyotroph Lateral Scler 2010;11:244-7.

10. Ahn SW, Kim SH, Kim JE, et al. Frontal assessment battery to evaluate frontal lobe dysfunction in ALS patients. Can J Neurol Sci 2011;38:242-6.

11. Floris $\mathrm{G}$, Borghero $\mathrm{G}$, Chiò $\mathrm{A}$, et al. Cognitive screening in patients with amyotrophic lateral sclerosis in early stages. Amyotroph Lateral Scler 2012;13:95-101.

12. Von Elm E, Altman DG, Egger M, et al., STROBE Initiative. Strengthening the Reporting of Observational Studies in
Epidemiology (STROBE) statement: guidelines for reporting observational studies. BMJ 2007;335:806-8.

13. Bossuyt PM, Reitsma JB, Bruns DE, et al., Standards for Reporting of Diagnostic Accuracy. The STARD statement for reporting studies of diagnostic accuracy: explanation and elaboration. Ann Intern Med 2003;138:W1-12.

14. Brooks BR, World Federation of Neurology Sub-Committee on Motor Neuron Diseases. El Escorial WFN criteria for the diagnosis of amyotrophic lateral sclerosis. J Neurol Sci 1994;124:965-1085.

15. American Psychiatric Association. Diagnostic and Statistical Manual of Mental Disorders (DSM IV-TR). 4th edn. Washington DC: American Psychiatric Association, 2000.

16. Magni E, Binetti G, Bianchetti A, et al. Mini-Mental State Examination: a normative study in Italian elderly population. Eur $\mathrm{J}$ Neurol 1996;3:1-5.

17. Cedarbaum JM, Stambler N, Malta E, et al. The ALSFRS-R: a revised ALS Functional Rating Scale that incorporates assessments of respiratory function. BDNF ALS Study Group (phase III). J Neurol Sci 1999;169:13-21.

18. Great Lakes ALS Study Group. A comparison of muscle strength testing techniques in amyotrophic lateral sclerosis. Neurology 2003;61:1503-7.

19. Czaplinski A, Yen AA, Appel SH. Forced vital capacity (FVC) as an indicator of survival and disease progression in an ALS clinic population. J Neurol Neurosurg Psychiatry 2006;77:390-2.

20. Smith A. Symbol Digit Modalities Test. Los Angeles, CA: Western Psychological Services, 1991.

21. Nocentini U, Giordano A, Di Vincenzo S, et al. The Symbol Digit Modalities Test-Oral Version: Italian normative data. Funct Neurol 2006;21:93-6.

22. Caffarra P, Vezzadini G, Dieci F, et al. Una versione abbreviata de test di Stroop: dati normativi nella popolazione Italiana. Riv Neurol 2002;12:111-15.

23. Benton AL, Hamsher K. Multilingual Aphasia Examination manual. lowa City: University of lowa, 1976.

24. Carlesimo GA, Caltagirone C, Gainotti G. The Mental deterioration Battery: normative data, diagnostic reliability and qualitative analyses of cognitive impairment. The Group for the Standardization of Mental Deterioration Battery. Eur Neurol 1996;36:378-84.

25. Beck AT, Steer RA, Brown GK. Manual for the Beck Depression Inventory-II. San Antonio, Texas: Psychological Corporation, 1996.

26. Muscarella LA, Barbano R, D'Angelo V, et al. Regulation of KEAP1 expression by promoter methylation in malignant gliomas and association with patient's outcome. Epigenetics 2011;6:317-25.

27. Hanley JA, McNeil BJ. The meaning and use of the area under a Receiver Operating Characteristic (ROC) curve. Radiology 1982;143:29-36.

28. Efron B, Tibshirani R. An Introduction to the Bootstrap. Boca Raton, FL: Chapman \& Hall/CRC, 1993.

29. Swets JA. Signal detection theory and ROC analysis in psychology and diagnostics: collected papers. Mahwah, NJ: Lawrence Erlbaum Associates, 1996.

30. Terada $\mathrm{T}$, Obi $\mathrm{T}$, Miyajima $\mathrm{H}$, et al. Assessing frontal lobe function in patients with amyotrophic lateral sclerosis by frontal assessment battery. Rinsho Shinkeigaku 2010;50:379-84.

31. Phukan J, Elamin M, Bede P, et al. The syndrome of cognitive impairment in amyotrophic lateral sclerosis: a population-based study. J Neurol Neurosurg Psychiatry 2012;83:102-8.

32. Atassi N, Cook A, Pineda CM, et al. Depression in amyotrophic lateral sclerosis. Amyotroph Lateral Scler 2011;12:109-12.

33. Kurt A, Nijboer F, Matuz T, et al. Depression and anxiety in individuals with amyotrophic lateral sclerosis: epidemiology and management. CNS Drugs 2007;21:279-91.

34. Wicks P, Abrahams S, Masi D, et al. Prevalence of depression in a 12-month consecutive sample of patients with ALS. Eur J Neurol 2007;14:993-1001.

35. Raaphorst J, Beeldman E, Jaeger B, et al. Is the Frontal Assessment Battery reliable in ALS patients? Amyotroph Lateral Scler Frontotemporal Degener 2013;14:73-4.

36. Osborne RA, Sekhon R, Johnston W, et al. Screening for frontal lobe and general cognitive impairment in patients with amyotrophic lateral sclerosis. J Neurol Sci 2014;336:191-6.

37. Lomen-Hoerth C, Anderson T, Miller B. The overlap of amyotrophic lateral sclerosis and frontotemporal dementia. Neurology 2002;59:1077-9.

38. Rhingholz GM, Appel SH, Bradshaw M, et al. Prevalence and patterns of cognitive impairment in sporadic ALS. Neurology 2005;65:586-90.

39. Lillo P, Savage S, Mioshi E, et al. Amyotrophic lateral sclerosis and frontotemporal dementia: a behavioural and cognitive continuum. Amyotroph Lateral Scler 2012;13:102-9. 
40. Renton AE, Majounie E, Waite A, et al. A hexanucleotide repeat expansion in C9ORF72 is the cause of chromosome 9p21-linked ALS-FTD. Neuron 2011;72:257-68.

41. DeJesus-Hernandez M, Mackenzie IR, Boeve BF, et al. Expanded GGGGCC hexanucleotide repeat in noncoding region of C9ORF72 causes chromosome 9p-linked FTD and ALS. Neuron 2011;72:245-56
42. Byrne $\mathrm{S}$, Elamin $\mathrm{M}$, Bede $\mathrm{P}$, et al. Cognitive and clinical characteristics of patients with amyotrophic lateral sclerosis carrying a C9orf72 repeat expansion: a population-based cohort study. Lancet Neurol 2012;11:232-40.

43. Abrahams S, Newton J, Niven E, et al. Screening for cognition and behaviour changes in ALS. Amyotroph Lateral Scler Frontotemporal Degener 2014;15:9-14. 